\title{
Rudolf Schlögl, Anwesende und Abwesende. Grundriss für eine Gesellschaftsgeschichte der Frühen Neuzeit
}

\author{
Falk Bretschneider
}

\section{OpenEdition}

\section{Journals}

Édition électronique

URL : http://journals.openedition.org/ifha/8731

DOI : 10.4000/ifha.8731

ISSN : 2198-8943

Éditeur

IFRA - Institut franco-allemand (sciences historiques et sociales)

Référence électronique

Falk Bretschneider, «Rudolf Schlögl, Anwesende und Abwesende. Grundriss für eine

Gesellschaftsgeschichte der Frühen Neuzeit », Revue de l'IFHA [En ligne], Date de recension, mis en ligne le 02 novembre 2017, consulté le 24 septembre 2020. URL : http://journals.openedition.org/ifha/8731 ; DOI : https://doi.org/10.4000/ifha.8731

Ce document a été généré automatiquement le 24 septembre 2020.

(C)IFHA 
Rudolf Schlögl, Anwesende und

Abwesende. Grundriss für eine Gesellschaftsgeschichte der Frühen

Neuzeit

Falk Bretschneider

\section{RÉFÉRENCE}

Rudolf Schlögl, Anwesende und Abwesende. Grundriss für eine Gesellschaftsgeschichte der Frühen Neuzeit, Konstanz: Konstanz University Press, 2014, 563 p., 30,90€ 
L'un des éléments les plus séduisants de la théorie luhmannienne est l'improbabilité d'une communication réussie. Puisque les choix des communicants, opérés à partir d'une variété a priori infinie de possibilités, s'inscrivent dans une contingence absolue, la communication a besoin de mécanismes stabilisateurs, parmi lesquels figurent ce que Niklas Luhmann appelle - avec le talent qui était le sien pour forger des notions aussi précises qu'intraduisibles - des Erwartungserwartungen. Autrement dit: des attentes que chaque partenaire de la communication adresse à l'autre en sachant que celui-ci fait de même. Ainsi, la communication ne peut réussir que si ces attentes réciproques sont remplies et confirmées, c'est-à-dire si ego correspond à l'image qu'alter se fait de lui (et vice

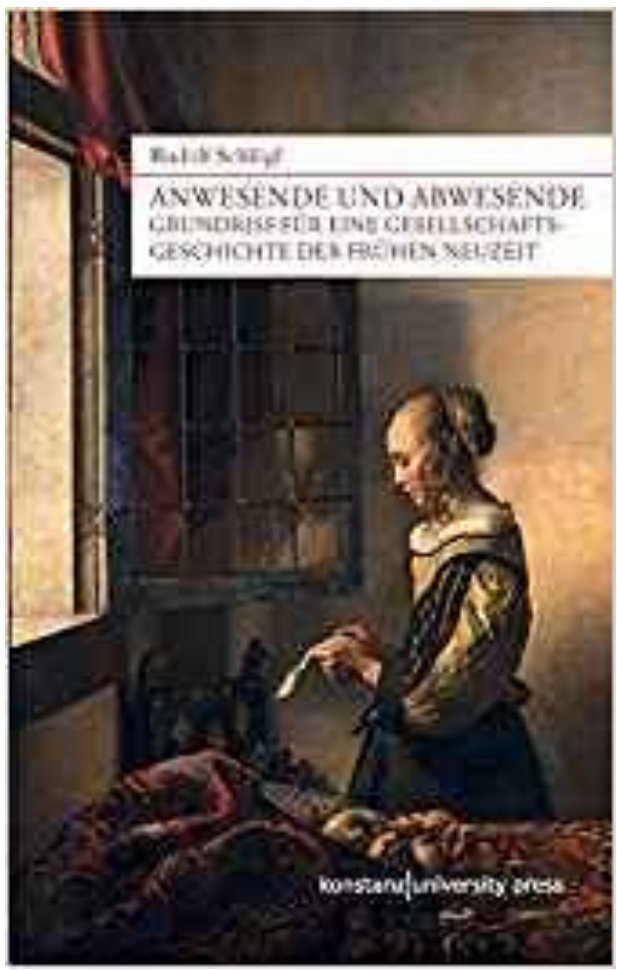
versa). D'où, par exemple, le rôle fondamental pour la vie en société de la politesse, qui n'est rien d'autre qu'une série d'attentes formalisées engendrant de la prévisibilité destinée à établir un rapport de communication réussi. Or, malgré ces opérations stabilisatrices, la vie est faite de communications ratées - et le livre de Rudolf Schlögl qui, justement, s'appuie sur la théorie communicationnelle forgée par le sociologue bielefeldien, en est un excellent exemple.

En se référant au titre qui annonce une " histoire de la société » moderne (autre salut adressé à l'université de Bielefeld, où l'école historique du même nom, dominée par Hans-Ulrich Wehler, avait développé dans les années 1970 et 1980 la version typiquement allemande de l'histoire structurelle), le lecteur historien pense pouvoir s'attendre à une synthèse d'histoire sociale moderne dont il est curieux de découvrir les contours. Il se rend cependant vite compte, dès les premières pages, que les attentes que l'auteur semble avoir par rapport à son lectorat diffèrent quelque peu. R. Schlögl s'adresse tout d'abord à un public intéressé par la théorie luhmanienne elle-même, qu'il souhaite non seulement élargir et développer pour en faire une référence utile aux historiens (encore frileux envers elle, dans leur majorité), mais qu'il veut également mettre à profit pour resusciter l'intérêt des sociologues envers l'histoire, largement disparu aujourd'hui. C'est donc un cas parfait d'attentes qui ne se correspondent pas, ce qui ne manque pas de provoquer quelques moments de consternation, notamment face au style d'un livre écrit dans un langage très abstrait, véritable défi lancé au lecteur même parfaitement germanophone, et dont le caractère abscons est encore accru par le fait que l'auteur ne s'est pas donné la peine d'expliquer les bases du cadre théorique auquel il se réfère: le lecteur, semble-t-il, est censé déjà les connaître. D'autres déceptions viennent s'ajouter à la première face à un ouvrage qui forme moins un inédit cohérent qu'un assemblage de textes déjà publiés ailleurs et seulement retravaillés, en partie, pour l'occasion. 
Dès lors, comment rendre justice à ce travail ? Car il le mérite, et pas seulement parce qu'il est le fruit de deux décennies d'intenses recherches de la part son auteur. L'objectif de R. Schlögl n'est rien moins que de donner un nouveau fondement à l'histoire moderne, en déplaçant l'outillage théorique et conceptuel des historiens d'une concentration sur les pratiques (dominante aujourd'hui, en France comme en Allemagne) vers un intérêt pour la communication. Plus large que celui de l'agir social (qui présuppose des attributions et donc de la communication), ce concept serait mieux à même de rendre compte de l'arbitraire de la formation de sens dans la société ainsi que de l'improbabilité et de la fragilité de l'ordre social. Il n'est pas nécessaire de partager cette vision (et l'auteur de ce compte-rendu ne le fait pas) pour concéder qu'elle représente une perspective digne d'intérêt par sa nouveauté. R. Schlögl s'appuie sur l'observation, sans doute juste, que l'historiographie de l'époque moderne n'a jusqu'à maintenant pas réussi à formuler une interprétation générale de l'évolution à la fin de laquelle est née la société contemporaine. Ses concepts-phares - la confessionnalisation, la disciplinarisation ou la juridification du social, pour n'en citer que quelques-uns - offrent des explications partielles, sans constituer une vision d'ensemble. R. Schlögl a pour ambition de proposer une telle perspective globale : la société qui, au début du XVIIe siècle, était marquée par des structures nécessitant de l'interaction, c'est-à-dire une communication en présence directe (soit des corps réels, soit par l'intermédiaire de symboles ou de rituels), évoluerait, à travers les possibilités offertes par les médias à distance que sont, par exemple, l'écriture, la presse ou l'argent, vers une société intégrant davantage de complexité et de réflexivité et n'exigeant plus la présence immédiate de ceux qui la constituent. Il s'agit donc d'expliquer la transformation d'une forme sociale qui a besoin du corps comme medium de sa communication vers une société qui dispose d'autres moyens, plus impersonnels, dissociés des situations concrètes et permettant de gérer la distance et l'absence, et qui remplace donc l'interaction par l'organisation.

Cette conception, qu'il serait trop long de détailler ici, d'autant que son auteur lui confère une densité impressionnante, répond indubitablement à l'intérêt actuel pour l'histoire des médias et leur rôle dans la structuration du social. Mais l'histoire que propose R. Schlögl n'est pas une histoire des médias. Bien au contraire, l'auteur réunit de nombreux thèmes chers à la recherche actuelle et les inscrit dans une approche d'histoire communicationnelle : l'honneur, l'espace, le corps, les rituels, mais aussi les formes de sens et les sémantiques que développe la société pour se décrire elle-même, etc. D'autres aspects qu'on aurait pu s'attendre à trouver dans une histoire qui ambitionne d'être celle d'une Gesellschaft toute entière, comme les différentes sphères du droit ou les rapports de genre, sont en revanche absents. En outre, dans ses parties davantage empiriques, le livre fait preuve d'une propension assez déroutante à trouver refuge auprès de "grands récits" finalement assez traditionnels: la formation de l'État, la naissance de l'administration moderne au sein des cours princières, les rapports entre Réforme et révolution médiatique... Certes, le lecteur ne peut pas ne pas saluer le brio de nombreuses analyses, même si le sentiment de n'en comprendre que la moitié est tenace. Mais quand il connaît un peu mieux les matières traitées, il lui arrive d'être surpris du caractère assez classique de l'interprétation. Ainsi, les parties sur l'émergence de l'État moderne ressemblent tout de même beaucoup aux récits traditionnels de la modernisation (faisant d'ailleurs largement écran aux particularités de l'évolution dans l'espace qui constitue le terrain de prédilection de l'auteur, à savoir le Saint-Empire). L'exemple est peut-être mal choisi, car R. Schlögl prétend écrire une 
nouvelle histoire de la société moderne, et non du politique. Mais justement: les références sont une fois de plus Castiglione, Bodin, Louis XIV ou Frédéric II - et non celles d'une histoire de la domination au ras du sol telle que toute une historiographie l'a écrite ces trente dernières années et qui aurait beaucoup de choses à dire, notamment sur la gestion des distances et les rapports de communication entre dominants et dominés.

Bref : le livre de R. Schlögl est sans doute un évènement, car il est impossible de l'ignorer. D'une certaine manière il enfonce même des portes ouvertes, car la différence entre présence et absence comme catégorie majeure de la structuration du social à l'époque moderne est si évidente qu'on peut s'étonner qu'elle n'ait pas été saisie plus tôt. C'est donc un livre important. Mais le lecteur s'y heurte davantage qu'il n'en profite. Non que les ambitions de l'auteur soient modestes ou ses propositions négligeables - bien au contraire. Mais il renoue avec une tradition historiographique malheureuse, surtout en Allemagne, celle qui place l'ardeur de la Theoriearbeit audessus de l'effort empirique consistant d'abord à expliquer le monde qui n'est plus à partir de ce qu'il a laissé (ce qui n'empêche en rien de le faire d'une manière théoriquement informée, comme le montrent les travaux de Barbara Stollberg-Rilinger, inspirés eux aussi de la théorie luhmanienne). L'auteur assume cette posture, c'est tout à son honneur, en revendiquant le statut de celui qui irrite - et nous savons bien qu'il faut parfois bousculer les codes de politesse pour faire bouger les mœurs. Il ne serait d'ailleurs pas non plus le premier à devenir ainsi le précurseur d'une nouvelle pensée. En attendant, il faut pourtant craindre que sa place soit celle du mal compris.

\section{INDEX}

Thèmes : Histoire sociale, Manuels/Ouvrages généraux/Outils de travail

Index chronologique : Période moderne

\section{AUTEURS}

\section{FALK BRETSCHNEIDER}

EHESS Paris 